\section{Pivoting from Laboratory to Remote Research Using the Online Game Foldit}

Zahra Tehrani

Purdue University, ztehrani@purdue.edu

\section{doi: $10.18833 /$ spur/4/4/7}

Honors College students at Purdue University are required to complete a capstone project as part of the curriculum. Many students experienced a disruption to their research plans in 2020 due to the COVID-19 pandemic. This prompted faculty to launch a place-based research initiative to recruit students to be onsite researchers from wherever they were located. As part of this program, the Foldit research group was created for students from biology-related majors. Developed by David Baker and colleagues at the University of Washington, Foldit is a free online game in which players compete to find a protein's best folded structure by solving 3D puzzles (Cooper et al. 2010). The starting structures of the puzzles are novel proteins predicted by automated algorithms to perform specific cellular functions. Calculating the most stable configuration of a protein from its amino acid sequence remains a grand challenge in computational biology. Humans, on the other hand, are more adept at spatial problem-solving than computers (Cooper et al. 2010). Foldit provides more than a dozen tools for players to manipulate or mutate structures in a way they believe will lead to the lowest energy conformation (highest game score). For this project, students were tasked with solving the coronavirus puzzles that aimed to solve the structure of protein binders against COVID-19's spike protein. The game also offers advanced puzzles for designing proteins from scratch. A total of 99 promising candidate proteins from Foldit players have been selected for laboratory testing so far (Bkoep 2020).

Foldit can only be downloaded onto laptop or desktop computers and requires at least $8 \mathrm{~Gb}$ of RAM. Students did not report any difference between working on and off campus, indicating that an internet speed of at least $25 \mathrm{Mbps}$ is sufficient. Four students participated in the project over two semesters - all had completed at least one semester of biochemistry or molecular biology. Because Foldit was designed for nonscientists and does not require any background knowledge in biology, a framework had to be developed around a set of learning outcomes for the project to have intellectual value for the students. First, a scientific foundation was needed for the design process. To this end, students were asked to write a literature review about the spike protein target. At the time, the protein's structure was an emerging research topic and had not been reviewed yet in the literature. Based on the faculty's analysis of the reviews, it was clear that all students gained an integrated understanding of the structure-function relationship of the spike protein and were able to identify critical gaps in the existing body of knowledge for future research. Second, to help guide students' design thinking, it was important for them to understand the thermodynamic forces driving protein folding. Most students who have taken courses in biochemistry or molecular biology are already familiar with the main principles; the game also provides a set of introductory puzzles that are helpful in this regard. To assess learning, students were required to submit critical reflections on their best-scoring solutions for two different puzzles. In the reflections, screenshots of the protein designs were presented with an in-depth analysis of the student's decision-making process (e.g., why did they move two beta-sheets closer together, and how did this affect their score?). Students were able to demonstrate critical thinking about their choices by making clear connections between specific folding concepts and the modifications made to the starting protein's structure.

The biggest challenge for students is to persist through all 32 introductory puzzles; students who skipped them reported feeling confused and frustrated with the game. Introductory puzzles introduce players to the mechanics of the game in incremental steps; therefore, it is essential for mentors to strongly encourage beginner players to complete all the tutorials before attempting the protein puzzles. Faculty may find it difficult defining the success of a design since the native structures of predicted proteins are unknown. One way to define success is the achievement of a predefined score or the student's global ranking (e.g., top 10). Faculty can also create private groups in Foldit to track student progress.

Foldit can serve as a free online option for undergraduates looking for research experiences when wet labs become inaccessible during times of constraints.

\section{References}

Bkoep. "Coronavirus Binder Designs Queued for Testing!" Foldit (blog). March 25, 2020. Accessed June 14, 2021. https:// fold.it/portal/node/2009216

Cooper, Seth, Firas Khatib, Adrien Treuille, Janos Barbero, Jeehyung Lee, Michael Beenen, Andrew Leaver-Fay, David Baker, and Zoran Popović. 2010. "Predicting Protein Structures with a Multiplayer Online Game." Nature 466: 756-760. doi: 10.1038/nature09304 